\title{
Robust Control of Nonlinear Systems with Hysteresis Based on Play-Like Operators
}

\author{
Jun $\mathrm{Fu}^{1}$, Wen-Fang $\mathrm{Xie}^{1}$, Shao-Ping Wang ${ }^{2}$ and Ying Jin ${ }^{3}$ \\ ${ }^{1}$ The Department of Mechanical \& Industrial Engineering \\ Concordia University \\ 2The Department of Mechatronic Control, Beihang University \\ ${ }^{3}$ State Key Laboratory of Integrated Automation of \\ Process Industry, Northeastern University \\ ${ }^{1}$ Canada \\ ${ }^{2,3}$ China
}

\section{Introduction}

Hysteresis phenomenon occurs in all smart material-based sensors and actuators, such as shape memory alloys, piezoceramics and magnetostrictive actuators ( $\mathrm{Su}$, et al, 2000; Fu, et al, 2007; Banks \& Smith, 2000; Tan \& Baras, 2004). When the hysteresis nonlinearity precedes a system plant, the nonlinearity usually causes the overall closed-loop systems to exhibit inaccuracies or oscillations, even leading to instability (Tao \& Kokotovic, 1995). This fact often makes the traditional control methods insufficient for precision requirement and even not be able to guarantee the basic requirement of system stability owing to the non-smooth and multi-value nonlinearities of the hysteresis (Tao \& Levis, 2001). Hence the control of nonlinear systems in presence of hysteresis nonlinearities is difficult and challenging ( $\mathrm{Fu}$, et al, 2007; Tan \& Baras, 2004).

Generally there are two ways to mitigate the effects of hysteresis. One is to construct an inverse operator of the considered hysteresis model to perform inversion compensation (Tan \& Baras, 2004; Tao \& Kokotovic, 1995; Tao \& Levis, 2001). The other is, without necessarily constructing an inverse, to fuse a suitable hysteresis model with available robust control techniques to mitigate the hysteretic effects ( $\mathrm{Su}$, et al, 2000; Fu, et al, 2007; Zhou, et al, 2004; Wen \& Zhou, 2007). The inversion compensation was pioneered in (Tao \& Kokotovic, 1995) and there are some other important results in (Tan \& Baras, 2005; Iyer, et al, 2005; Tan \& Bennani, 2008). However, most of these results were achieved only at actuator component level without allowing for the overall dynamic systems with actuator hysteresis nonlinearities. Essentially, constructing inverse operator relies on the phenomenological model (such as Preisach models) and influences strongly the practical application of the design concept (Su, et al, 2000). Because of multi-valued and non-smoothness feature of hysteresis, those methods are often complicated, computationally costly and possess strong sensitivity of the model parameters to unknown measurement errors. These issues are directly linked to the difficulties of guaranteeing the stability of systems except for certain special cases (Tao \& Kokotovic, 1995). For the methods to mitigate hysteretic effects without constructing the inverse, there are two main challenges involved in this idea. One challenge is that very few hysteresis models 
are suitable to be fused with available robust adaptive control techniques. And the other is how to fuse the suitable hysteresis model with available control techniques to guarantee the stability of the dynamics systems (Su, et al, 2000). Hence it is usually difficult to construct new suitable hysteresis models to be fused into control plants, and to explore new control techniques to mitigate the effects of hysteresis and to ensure the system stability, without necessarily constructing the hysteresis inverse.

Noticing the above challenges, we first construct a hysteresis model using play-like operators, in a similar way to L. Prandtl's construction of the Prandtl-Ishilinskii model using play operators (Brokate \& Sprekels, 1996), and thus name it Prandtl-Ishilinskii-Like model. Because the play-like operator in (Ekanayake \& Iyer, 2008) is a generalization of the backlash-like operator in (Su, et al, 2000), the Prandtl-Ishilinskii-Like model is a subclass of SSSL-PKP hysteresis model (Ekanayake \& Iyer, 2008). Then, the development of two robust adaptive control schemes to mitigate the hysteresis avoids constructing a hysteresis inverse. The new methods not only can perform global stabilization and tracking tasks of the dynamic nonlinear systems, but also can derive transient performance in terms of $L_{2}$ norm of tracking error as an explicit function of design parameters, which allows designers to meet the desired performance requirement by tuning the design parameters in an explicit way.

The main contributions in this chapter are highlighted as follows:

i. A new hysteresis model is constructed, where the play-like operators developed in (Ekanayake \& Iyer, 2008) play a role of building blocks. From a standpoint of categories of hysteresis models, this class of hysteresis models is a subclass of SSSL-PKP hysteresis models. It provides a possibility to mitigate the effects of hysteresis without necessarily constructing an inverse, which is the unique feature of this subclass model identified from the SSSL-PKP hysteresis model of general class in the literature;

ii. A challenge is addressed to fuse a suitable hysteresis model with available robust adaptive techniques to mitigate the effects of hysteresis without constructing a complicated inverse operator of the hysteresis model;

iii. Two backstepping schemes are proposed to accomplish robust adaptive control tasks for a class of nonlinear systems preceded by the Prandtl-Ishilinskii-Like models. Such control schemes not only ensure the stabilization and tracking of the hysteretic dynamic nonlinear systems, but also derive the transient performance in terms of $L_{2}$ norm of tracking error as an explicit function of design parameters.

The organization of this chapter is as follows. Section 2 gives the problem statement. In Section 3, we will construct Prandtl-Ishlinshii-Like model and explore its properties. The details about two control schemes for the nonlinear systems preceded by Prandtl-IshlinshiiLike model proposed in Section 3 are presented in Section 4. Simulation results are given in Section 5. Section 6 concludes this paper with some brief remarks.

\section{Problem statement}

Consider a controlled system consisting of a nonlinear plant preceded by an actuator with hysteresis nonlinearity, that is, the hysteresis is presented as an input to the nonlinear plant. The hysteresis is denoted as an operator

$$
w(t)=P[v](t)
$$


with $v(t)$ as the input and $w(t)$ as the output. The operator $P[v]$ will be constructed in detail in next section. The nonlinear dynamic system being preceded by the previous hysteresis is described in the canonical form as

$$
x^{(n)}(t)+\sum_{i=1}^{k} a_{i} Y_{i}\left(x(t), \dot{x}(t), \cdots, x^{(n-1)}(t)\right)=b w(t)
$$

where $Y_{i}$ are known continuous, linear or nonlinear function. Parameters $a_{i}$ and control gain $b$ are unknown constants. It is a common assumption that the sign of $b$ is known. Without losing generality, we assume $b$ is greater than zero. It should be noted that more general classes of nonlinear systems can be transformed into this structure (Isidori, 1989).

The control objective is to design controller $v(t)$ in (1), as shown in Figure 1, to render the plant state $x(t)$ to track a specified desired trajectory $x_{d}(t)$, i.e., $x(t) \rightarrow x_{d}(t)$ as $t \rightarrow \infty$.

Throughout this paper the following assumption is made.

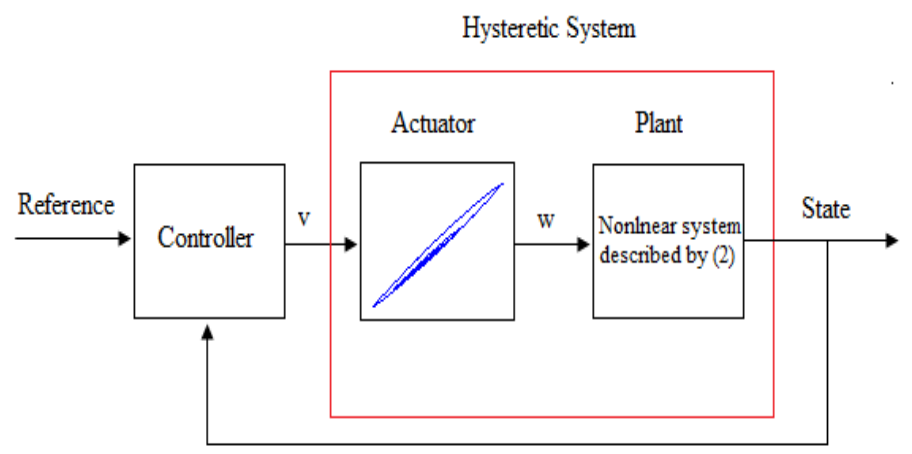

Fig. 1. Configuration of the hysteretic system

Assumption: The desired trajectory $X_{d}=\left[x_{d}, \dot{x}_{d}, \cdots, x_{d}^{(n-1)}\right]^{T}$ is continuous. Furthermore, $\left[X_{d}^{T}, x_{d}^{(n)}\right]^{T} \in \Omega_{d} \subset R^{n+1}$ with $\Omega_{d}$ being a compact set.

\section{PrandtI-Ishlinskii-Like model}

In this section, we will first recall the backlash-like operator ( $\mathrm{Su}$, et al, 2000) which will serve as elementary hysteresis operator, in other words, the backlash-like operator will play a role of building blocks, then will show how the new hysteresis will be constructed by using the backlash-like operator and explore its some useful properties of this model.

\subsection{Backlash-like operator}

In 2000, Su et al proposed a continuous-time dynamic model to describe a class of backlashlike hysteresis, as given by

$$
\frac{d F}{d t}=\alpha\left|\frac{d v}{d t}\right|(c v-F)+B_{1} \frac{d v}{d t}
$$


where $\alpha, c$, and $B_{1}$ are constants, satisfying $c>B_{1}$.

The solution of (3) can be solved explicitly for piecewise monotone $v$ as follows

$$
F(t)=c v(t)+\left[F_{0}-c v_{0}\right] e^{-\alpha\left(v-v_{0}\right) \operatorname{sgn} \dot{v}}+e^{-\alpha v \operatorname{sgn} \dot{v}} \int_{v_{0}}^{v}\left[B_{1}-c\right] e^{\alpha \zeta(\operatorname{sgn} \dot{v})} d \zeta
$$

for $\dot{v}$ constant and $w\left(v_{0}\right)=w_{0}$. Equation (4) can also be rewritten as

$$
F(t)= \begin{cases}c v(t)+\left[F_{0}-c v_{0}\right] e^{-\alpha\left(v-v_{0}\right)}+e^{-\alpha v} \frac{B_{1}-c}{\alpha}\left(e^{\alpha v}-e^{\alpha v_{0}}\right), & \dot{v}>0 \\ c v(t)+\left[F_{0}-c v_{0}\right] e^{\alpha\left(v-v_{0}\right)}+e^{\alpha v} \frac{B_{1}-c}{-\alpha}\left(e^{-\alpha v}-e^{-\alpha v_{0}}\right), & \dot{v}<0\end{cases}
$$

It is worth to note that

$$
\begin{aligned}
& \lim _{v \rightarrow+\infty}(F(v)-c v)=-\frac{c-B_{1}}{\alpha} \\
& \lim _{v \rightarrow-\infty}(F(v)-c v)=\frac{c-B_{1}}{\alpha}
\end{aligned}
$$

Hence, solution $F(t)$ exponentially converges the output of a play operator with threshold $r=\frac{c-B_{1}}{\alpha}$ and switches between lines $c v+\frac{c-B_{1}}{\alpha}$ and $c v-\frac{c-B_{1}}{\alpha}$. We will construct a new Prandtl-Ishilinskii-Like model by using the above backlash-like model in next subsection, similar to the construction of the well-known Prandtl-Ishilinskii model from play operators, which is our motivation behind the construction of this new model indeed.

\subsection{Prandtl-Ishilinskii-Like model}

We now ready to construct Prandtl-Ishilinskii-Like model through a weighted superposition of elementary backlash-like operator $F_{r}[v](t)$, in a similar way as L. Prandtl (Brokate \& Sprekels, 1996) constructed Prandtl-Ishilinskii model by using play operators.

Keep $r=\frac{c-B_{1}}{\alpha}$ in mind and, without losing generality, set $F(v(0)=0)=0$ and $c=1$, we rewrite equation (5) as

$$
F_{r}(t)=\left\{\begin{array}{cc}
v(t)+r-r e^{-\frac{-\left(1-B_{1}\right)}{r} v}, \quad & \dot{v}>0 \\
v(t)-r+r e^{\frac{1-B_{1}}{r} v}, & \dot{v}<0
\end{array}\right.
$$

where $r$ is the threshold of the backlash-like operator.

To this end, we construct the Prandtl-Ishilinskii-Like model by

$$
w(t)=\int_{0}^{R} p(r) F_{r}[v](t) d r
$$


where $p(r)$ is a given continuous density function, satisfying $p(r) \geq 0$ with $\int_{0}^{\infty} p(r) d r<+\infty$, and is expected to be identified from experimental data (Krasnoskl'skill \& Pokrovskill, 1983; Brokate \& Sprekels, 1996). Since the density function $p(r)$ vanishes for large values of $r$, the choice of $R=+\infty$ as the upper limit of integration in the literature is just a matter of convenience (Brokate \& Sprekels, 1996).

Inserting (7) into (8) yields

$$
w[v](t)= \begin{cases}\int_{0}^{R} p(r) d r \cdot v(t)+\int_{0}^{R} p(r)\left(r-r e^{\frac{-\left(1-B_{1}\right)}{r} v}\right) d r, & \dot{v}>0 \\ \int_{0}^{R} p(r) d r \cdot v(t)+\int_{0}^{R} p(r)\left(-r+r e^{-\frac{1-B_{1}}{r} v}\right) d r, & \dot{v}<0\end{cases}
$$

the hysteresis (9) can be expressed as

$$
w(t)=p_{0} v+ \begin{cases}\int_{0}^{R} p(r)\left(r-r e^{\frac{-\left(1-B_{1}\right)}{r} v}\right) d r, & \dot{v}>0 \\ \int_{0}^{R} p(r)\left(-r+r e^{-\frac{1-B_{1}}{r} v}\right) d r, & \dot{v}<0\end{cases}
$$

where $p_{0}=\int_{0}^{R} p(r) d r$ is a constant which depends on the density function $p(r)$.

Property 1: Let

$$
d[v](t)= \begin{cases}\int_{0}^{R} p(r)\left(r-r e^{\frac{-\left(1-B_{1}\right)}{r} v}\right) d r, & \dot{v}>0 \\ \int_{0}^{R} p(r)\left(-r+r e^{-\frac{1-B_{1}}{r} v}\right) d r, & \dot{v}<0\end{cases}
$$

satisfying $p(r) \geq 0$ with $\int_{0}^{\infty} p(r) d r<+\infty$, then for any $v(t) \in C_{p m}\left(t_{0}, \infty\right)$, there exists a constant $M \geq 0$ such that $|d[v](t)| \leq M$.

Proof: since (7) can be rewritten as $F_{r}(t)=v(t)+R(r, v)$ where

$$
R(r, v)=\left\{\begin{array}{cc}
r-r e^{-\frac{-\left(1-B_{1}\right)}{r} v}, & \dot{v}>0 \\
-r+r e^{\frac{1-B_{1}}{r} v}, & \dot{v}<0
\end{array}\right.
$$

Based on the analysis in (Su, et al, 2000), for each fixed $r \in(0, R)$, it is always possible there exists a positive constant $M_{1}$, such that $|R(r, v)| \leq M_{1}$. Hence

$$
d[v](t)=\int_{0}^{R} p(r) R(r, v) d r \leq \int_{0}^{R} p(r)|R(r, v)| d r \leq M_{1} \int_{0}^{R} p(r) d r
$$


By the definition of $p(r)$, one can conclude that $M=M_{1} \int_{0}^{R} p(r) d r$.

Property 2: the Prandtl-Ishilinskii-Like model constructed by (9) is rate-independent.

Proof: Following (Brokate \& Sprekels, 1996), we let $\sigma:\left[0, t_{E}\right] \rightarrow\left[0, t_{E}\right]$ satisfying $\sigma(0)=0$ and $\sigma\left(t_{E}\right)=t_{E}$ be a continuous increasing function, i.e. $\sigma(\cdot)$ is an admissible time transformation and define $w_{f}\left[v_{t}\right]$ satisfying $w_{f}\left[v_{t}\right]=w[v](t), t \in\left[0, t_{E}\right]$ and $v \in M_{p m}\left[0, t_{E}\right]$ where $v_{t}$ represents the truncation of $v$ at $t$, defined by $v_{t}(\tau)=v(\tau)$ for $0 \leq \tau \leq t$ and $v_{t}(\tau)=v(t)$ for $t \leq \tau \leq t_{E}$, and $w[v](t)$ constructed by (9). For the model (9), we can easily have

$$
w[v \circ \sigma](t)=w_{f}\left[(v \circ \sigma)_{t}\right]=w_{f}\left[v_{\sigma(t)} \circ \sigma\right]=w_{f}\left[v_{\sigma(t)}\right]=w[v](\sigma(t))=w[v](t) \circ \sigma(t)
$$

Hence for all admissible time transformation $\sigma(\cdot)$, according to the definition 2.2.1 in (Brokate \& Sprekels, 1996), the model constructed by (9) is rate-independent.

Property 3: the Prandtl-Ishilinskii-Like model constructed by (9) has the Volterra property. Proof: it is obvious whenever $v, \bar{v} \in M_{p m}\left[0, t_{E}\right]$ and $t \in\left[0, t_{E}\right]$, then $v_{t}=\bar{v}_{t}$ implies that $(w[v])_{t}=(w[\bar{v}])_{t}$, so, according to (Brokate \& Sprekels, 1996, Page 37), the model (8) has Volterra property.

Lemma 1: If a functional $w: C_{p m}\left[0, t_{E}\right] \rightarrow \operatorname{Map}\left(\left[0, t_{E}\right]\right)$ has both rate independence property and Volterra property, then $w$ is a hysteresis operator (Brokate \& Sprekels, 1996).

Proposition 1: the Prandtl-Ishilinskii-Like model constructed by (9) is a hysteresis operator. Proof: From the Properties 1, 2 and Lemma 1, the Prandtl-Ishilinskii-Like model (9) is a hysteresis model.

Remark 1: It should be mentioned that Prandtl-Ishilinskii model is a weighted superposition of play operator, i.e. play operator is the hysteron (Krasnoskl'skill \& Pokrovskill, 1983), and that backlash-like operator can be viewed as a play-like operator from a 1st order differential equation (Ekanayake \& Iyer, 2008). Hence, the model (8) is, with a litter abuse terminology, named Prandtl-Ishilinskii-Like model. As an illustration, Figure 2 shows $w(t)$ generated by (9), with $p(r)=e^{-6.7(0.1 r-1)^{2}} \quad r \in(0,50], \quad B_{1}=0.505$, and input $v(t)=7 \sin (4 t) /(1+t)$, with $F(v(0)=0)=0$.

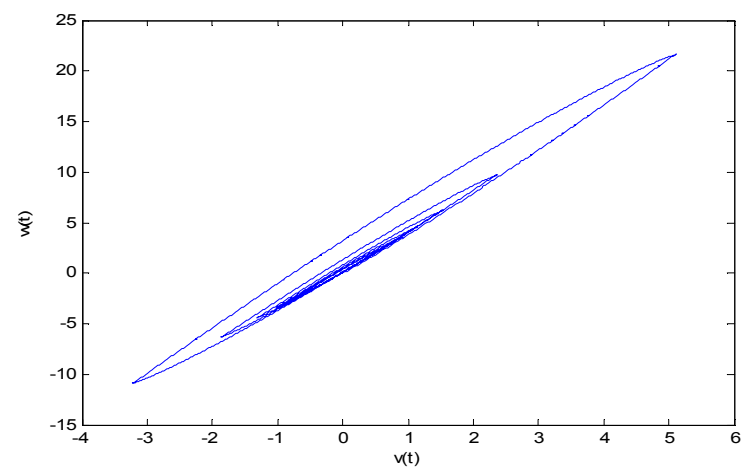

Fig. 2. Prandtl-Ishlinskii-Like Hysteresis curves given by (10) 
Remark 2: From another point of an alternative one-parametric representation of Preisach operator (Krejci, 1996), the Prandtl-Ishilinskii-Like model falls into PKP-type operator (Ekanayake \& Iyer, 2008), as Prandtl-Ishilinskii model into Preisach model. As a preliminary step, in the paper we explore the properties of this model and its potential to facilitate control when a system is preceded by this kind of hysteresis model, which will be demonstrated in the next section. Regarding hysteresis phenomena in which kind of smart actuator this model could characterize, it is still unclear. The future work will focus on, which is beyond of the scope of this paper.

To this end, we can rewrite (9) into

$$
w(t)=p_{0} v+d[v](t)
$$

where $p_{0}=\int_{0}^{R} p(r) d r$ and $d[v](t)$ is defined by (11).

Remark 3: It should be note that (10) decomposes the hysteresis behavior into two terms. The first term describes the linear reversible part, while the second term describes the nonlinear hysteretic behavior. This decomposition is crucial (Su, et al, 2000, Fu, et al, 2007) since it facilitates the utilization of the currently available control techniques for the controller design, which will be clear in next section.

\section{Adaptive control design}

From (10) and Proposition 1 we see that the signal $w(t)$ is expressed as a linear function of input signal $v(t)$ plus a bounded term. Using the hysteresis model of (10), the nonlinear system dynamics described by (2), can be re-expressed as

$$
\begin{aligned}
\dot{x}_{1}= & x_{2} \\
\vdots & \\
\dot{x}_{n-1}= & x_{n} \\
\dot{x}_{n}= & -\sum_{i=1}^{k} a_{i} Y_{i}\left(x_{1}(t), x_{2}(t), \cdots, x_{n}(t)\right) \\
& +b\left\{p_{0} v(t)-d[v](t)\right\} \\
= & \mathbf{a}^{T} Y+b_{p} v(t)-d_{b}[v]
\end{aligned}
$$

where $x_{1}(t)=x(t), x_{2}(t)=\dot{x}(t), \cdots, x_{n}(t)=x^{(n-1)}(t), \mathbf{a}=\left[-a_{1},-a_{2}, \cdots,-a_{k}\right]^{T}$, and $b_{p}=b p_{0}$ $Y=\left[Y_{1}, Y_{2}, \cdots, Y_{k}\right]^{T}$, and $d_{b}[v](t)=b d[v](t)$.

Before presenting the adaptive control design using the backstepping technique in (Krisic, et al, 1995) to achieve the desired control objectives, we make the following change of coordinates:

$$
\begin{aligned}
& z_{1}=x_{1}-x_{d} \\
& z_{i}=x_{i}-x_{d}{ }^{(i-1)}-\alpha_{i-1}, \quad i=2,3, \cdots, n
\end{aligned}
$$

Where $\alpha_{i-1}$ is the virtual controller in the $i$ th step and will be determined later. In the following, we give two control schemes. In Scheme I, the controller is discontinuous; the other is continuous in Scheme II. 


\section{Scheme I}

In what follows, the robust adaptive control law will be developed for Scheme I.

First, we give the following definitions

$$
\begin{aligned}
& \tilde{\mathbf{a}}(t)=\mathbf{a}-\hat{\mathbf{a}}(t) \\
& \tilde{\phi}(t)=\phi-\hat{\phi}(t) \\
& M(t)=M-\hat{M}(t)
\end{aligned}
$$

where $\hat{\mathbf{a}}$ is an estimate of $\mathbf{a}, \hat{\phi}$ is an estimate of $\phi$, which is defined as $\phi:=\frac{1}{b_{p}}$, and $\hat{M}$ is an
estimate of $M$. Given the plant and the hysteresis model subject to the assumption above, we propose the following control law

$$
\begin{aligned}
& v(t)=\hat{\phi}(t) v_{1}(t) \\
& v_{1}(t)=-c_{n} z_{n}-z_{n-1}-\hat{\mathbf{a}}^{T} Y-\operatorname{sgn}\left(z_{n}\right) \hat{D}+x_{d}^{(n)}+\dot{\alpha}_{n-1} \\
& \dot{\hat{\phi}}(t)=-\eta v_{1}(t) z_{n} \\
& \dot{\hat{\mathbf{a}}}(t)=\Gamma Y z_{n} \\
& \dot{M}(t)=\gamma\left|z_{n}\right|
\end{aligned}
$$

where $c_{n}, \eta$, and $\gamma$ are positive design parameters, and $\Gamma$ is a positive-definite matrix. These parameters can provide a certain degree of freedom to determine the rates of the adaptations. And $\alpha_{n-1}$ and the implicit $\alpha_{i-1}, i=2,3, \cdots, n-1$ in (16) will be designed in the proof of the following theorem for stability analysis.

The stability of the closed-loop system described in (13) and (16) is established as:

Theorem 1: For the plant given in (2) with the hysteresis (8), subject to Assumption 1, the robust adaptive controller specified by (16) ensures the following statements hold.

i. The resulting closed-loop system (2) and (8) is globally stable in the sense that all the signals of the closed-loop system ultimately bounded;

ii. The asymptotic tracking is achieved, i.e., $\lim _{t \rightarrow \infty}\left[x(t)-x_{d}(t)\right]=0$;

iii. The transient tracking error can be explicitly specified by

$$
\left\|x(t)-x_{d}(t)\right\|_{2} \leq \sqrt{\frac{\left(\frac{1}{2} \tilde{\mathbf{a}}(0)^{T} \Gamma^{-1} \tilde{\mathbf{a}}(0)+\frac{b_{p}}{2 \eta} \tilde{\phi}(0)^{2}+\frac{1}{2 \gamma} \tilde{M}(0)^{2}\right)}{c_{1}}}
$$

Proof: we will use a standard backstepping technique to prove the statements in a systematically way as follows:

Step 1: The time derivative of $z_{1}$ can be computed as

$$
\dot{z}_{1}=z_{2}+\alpha_{1}
$$

The virtual control $\alpha_{1}$ can be designed as 


$$
\alpha_{1}=-c_{1} z_{1}
$$

where $c_{1}$ is a positive design parameter.

Hence, we can get the first equation of tracking error

$$
\dot{z}_{1}=z_{2}-c_{1} z_{1}
$$

Step 2: Differentiating $z_{2}$ gives

$$
\dot{z}_{2}=z_{3}+\alpha_{2}-\dot{\alpha}_{1}
$$

The virtual control $\alpha_{2}$ can be designed as

$$
\alpha_{2}=-c_{2} z_{2}-z_{1}+\dot{\alpha}_{1}
$$

Hence the dynamics is

$$
\dot{z}_{2}=-c_{2} z_{2}-z_{1}+z_{3}
$$

Following this procedure step by step, we can derive the dynamics of the rest of states until the real control appears.

Step n: the $\mathrm{n}$-th dynamics are given by

$$
\dot{z}_{n}=b_{p} v(t)+a^{T} Y-x_{d}^{(n)}-\dot{\alpha}_{n-1}+d_{b}[v](t)
$$

We design the real control as follows:

$$
\begin{aligned}
& v(t)=\hat{\phi}(t) v_{1}(t) \\
& v_{1}(t)=-c_{n} z_{n}-z_{n-1}-\hat{\mathbf{a}}^{T} Y-\operatorname{sgn}\left(z_{n}\right) \hat{M}+x_{d}^{(n)}+\dot{\alpha}_{n-1} \\
& \dot{\hat{\phi}}(t)=-\eta v_{1}(t) z_{n} \\
& \dot{\hat{\mathbf{a}}}(t)=\Gamma Y z_{n} \\
& \dot{M}(t)=\gamma\left|z_{n}\right|
\end{aligned}
$$

Note that $b_{p} v(t)$ in (19) can be expressed as

$$
b_{p} v(t)=b_{p} \hat{\phi}(t) v_{1}(t)=v_{1}(t)-b_{p} \tilde{\phi}(t) v_{1}(t)
$$

Hence, we obtain

$$
\dot{z}_{n}=-c_{n} z_{n}-z_{n-1}-\hat{\mathbf{a}}^{T} Y-\operatorname{sgn}\left(z_{n}\right) \hat{M}+d_{b}[v](t)-b_{p} \tilde{\phi}(t) v_{1}(t)
$$

To this end, we defend the candidate Lyapunov function as

$$
V=\sum_{i=1}^{n} \frac{1}{2} z_{i}^{2}+\frac{1}{2} \tilde{\mathbf{a}}^{T} \Gamma^{-1} \tilde{\mathbf{a}}+\frac{b_{p}}{2 \eta} \tilde{\phi}^{2}+\frac{1}{2 \gamma} \tilde{M}^{2}
$$

The derivative $\dot{V}$ is given by 


$$
\begin{aligned}
\dot{V} & =\sum_{i=1}^{n} z_{i} \dot{z}_{i}+\tilde{\mathbf{a}}^{T} \Gamma^{-1} \dot{\tilde{\mathbf{a}}}+\frac{b_{p}}{\eta} \tilde{\phi} \dot{\tilde{\phi}}+\frac{1}{\gamma} \tilde{M} \dot{\tilde{M}} \\
& \leq \sum_{i=1}^{n} c_{i} z_{i}^{2}+\tilde{\mathbf{a}}^{T} \Gamma^{-1}\left(\Gamma Y z_{n}-\dot{\hat{\mathbf{a}}}\right)-\frac{b_{p}}{\eta} \tilde{\phi}\left(\eta v_{1} z_{n}+\dot{\hat{\phi}}\right)-\left|z_{n}\right| \hat{M}+\left|z_{n}\right|\left|d_{b}[v](t)\right|+\frac{1}{\gamma} \tilde{M} \dot{\tilde{M}} \\
& \leq-\sum_{i=1}^{n} c_{i} z_{i}^{2}+\tilde{\mathbf{a}}^{T} \Gamma^{-1}\left(\Gamma Y z_{n}-\dot{\hat{\mathbf{a}}}\right)-\frac{b_{p}}{\eta} \tilde{\phi}\left(\eta v_{1} z_{n}+\dot{\hat{\phi}}\right)+\frac{1}{\gamma} \tilde{M}\left(\gamma\left|z_{n}\right|-\dot{\hat{M}}\right) \\
& =-\sum_{i=1}^{n} c_{i} z_{i}^{2}
\end{aligned}
$$

Equations (22) and (23) imply that $V$ is nonincreasing. Hence, the boundedness of the variables $z_{1}, z_{2}, \cdots, z_{n}, \hat{\phi}, \hat{\mathbf{a}}, \hat{M}$ are ensured. By applying the LaSalle-Yoshizawa Theorem (Krisic, et al, 1995, Theorem 2.1), if further follows that $z_{i} \rightarrow 0, i=1,2, \cdots, n$ as time goes to infinity, which implies $\lim _{t \rightarrow \infty}\left[x(t)-x_{d}(t)\right]=0$.

We can prove the third statement of Theorem 1 in the following way.

From (23), we know

$$
\left\|z_{1}\right\|_{2}^{2}=\int_{0}^{\infty}\left|z_{1}(s)\right|^{2} d s \leq \frac{V(0)-V(\infty)}{c_{1}} \leq \frac{V(0)}{c_{1}}
$$

Noticing $V(0)=\frac{1}{2} \tilde{\mathbf{a}}(0)^{T} \Gamma^{-1} \tilde{\mathbf{a}}(0)+\frac{b_{p}}{2 \eta} \tilde{\phi}(0)^{2}+\frac{1}{2 \gamma} \tilde{M}(0)^{2}$ after setting $z_{i}(0)=0, i=1,2, \cdots, n$, hence

$$
\left\|x(t)-x_{d}(t)\right\|_{2} \leq \sqrt{\frac{\left(\frac{1}{2} \tilde{\mathbf{a}}(0)^{T} \Gamma^{-1} \tilde{\mathbf{a}}(0)+\frac{b_{p}}{2 \eta} \tilde{\varphi}(0)^{2}+\frac{1}{2 \gamma} \tilde{M}(0)^{2}\right)}{c_{1}}}
$$

Remark 4: From (24), we know that the transient performance in a computable explicit form depends on the design parameters $\eta, \gamma, c_{1}$ and on the initial estimate errors $\tilde{\mathbf{a}}(0), \tilde{\phi}(0) \tilde{M}(0)$, which gives designers enough tuning freedom for transient performance.

\section{Scheme II}

In the control scheme above, we notice that in the controller, there is $\operatorname{sgn}\left(z_{n}\right)$ introduced in the design process, which makes the controller discontinuous and this may cause undesirable chattering. An alternative smooth scheme is proposed to avoid possible chattering with resort to the definition of continuous sign function (Zhou et al, 2004).

First, the definition of $s g_{i}\left(z_{i}\right)$ is introduced as follows:

$$
s g_{i}\left(z_{i}\right)= \begin{cases}\frac{z_{i}}{\left|z_{i}\right|^{\prime}} & \left|z_{i}\right| \geq \delta_{i} \\ \frac{z_{i}}{\left|z_{i}\right|+\left(\delta_{i}^{2}-z_{i}^{2}\right)^{n-i+2}} & \left|z_{i}\right|<\delta_{i}\end{cases}
$$


where design parameter $\delta_{i}(i=1, \cdots, n)$ is positive. It can be known that $s g_{i}\left(z_{i}\right)$ has $(n-i+2)$ th order derivatives.

Hence we have

$$
s g_{i}\left(z_{i}\right) f_{i}\left(z_{i}\right)=\left\{\begin{array}{cc}
1, & z_{i} \geq \delta_{i} \\
0, & \left|z_{i}\right|<\delta_{i} \\
-1, & z_{i} \leq-\delta_{i}
\end{array}\right.
$$

where

$$
f_{i}\left(z_{i}\right)= \begin{cases}1, & \left|z_{i}\right| \geq \delta_{i} \\ 0, & \left|z_{i}\right|<\delta_{i}\end{cases}
$$

Given the plant and the hysteresis model subject to the assumption above, we propose the following continuous controller as follows:

$$
\begin{aligned}
& v(t)=\hat{\phi}(t) v_{1}(t) \\
& v_{1}(t)=-\left(c_{n}+1\right)\left(\left|z_{n}\right|-\delta_{n}\right) s g_{n}\left(z_{n}\right)-\hat{\mathbf{a}}^{T} Y-s g_{n}\left(z_{n}\right) \hat{M}+x_{d}^{(n)}+\dot{\alpha}_{n-1} \\
& \dot{\hat{\phi}}(t)=-\eta v_{1}(t)\left(\left|z_{n}\right|-\delta_{n}\right) f_{n} s g_{n}\left(z_{n}\right) \\
& \dot{\hat{\mathbf{a}}}(t)=\Gamma Y\left(\left|z_{n}\right|-\delta_{n}\right) f_{n} s g_{n}\left(z_{n}\right) \\
& \dot{M}(t)=\gamma\left(\left|z_{n}\right|-\delta_{n}\right) f_{n}
\end{aligned}
$$

where, similarly as Control Scheme $1, c_{n}, \eta$, and $\gamma$ are positive design parameters, and $\Gamma$ is a positive-definite matrix, and $\alpha_{n-1}$ and the implicit $\alpha_{i-1}, i=2,3, \cdots, n-1$ in (26) will be designed in the proof of the following theorem for stability analysis.

Theorem 2: For the plant given in (2) with the hysteresis (8), subject to Assumption 1, the robust adaptive controller specified by (26) ensures the following statements hold.

i. The resulting closed-loop system (2) and (8) is globally stable in the sense that all the signals of the closed-loop system ultimately bounded;

ii. The tracking error can asymptotically reach to $\delta_{1}$, i.e., $\lim _{t \rightarrow \infty}\left[x(t)-x_{d}(t)\right]=\delta_{1}$;

iii. The transient tracking error can be explicitly specified by

$$
\left\|x(t)-x_{d}(t)\right\|_{2} \leq \delta_{1}+\frac{1}{c_{1}^{2 n}}\left(\frac{1}{2} \tilde{\mathbf{a}}(0)^{T} \Gamma^{-1} \tilde{\mathbf{a}}(0)+\frac{b_{p}}{2 \eta} \tilde{\phi}(0)^{2}+\frac{1}{2 \gamma} \tilde{M}(0)^{2}\right)^{1 / 2 n}
$$

Proof: To guarantee the differentiability of the resultant functions, $z_{i}^{2}$ in the Lyaounov functions will be replaced by $\left(\left|z_{i}\right|-\delta_{i}\right)^{n-i+2} f_{i}$ in Section 3.1 and $z_{i}$ in the design procedure detailed below will be replaced by $\left(\left|z_{i}\right|-\delta_{i}\right)^{n-i+1} s g_{i}$ as did in (Zhou et al, 2004).

Step 1: We choose a positive-definition function $V_{1}$ as

$$
V_{1}=\frac{1}{n+1}\left(\left|z_{1}\right|-\delta_{1}\right)^{n+1} f_{1}\left(z_{1}\right),
$$


and design virtual controller $\alpha_{1}$ as

$$
\alpha_{1}=-\left(c_{1}+k\right)\left(\left|z_{1}\right|-\delta_{1}\right)^{n} s g_{1}\left(z_{1}\right)-\left(\delta_{2}+1\right) s g_{1}\left(z_{1}\right)
$$

with constant $k$ satisfying $0<k \leq \frac{1}{4}$ and a positive design parameter $c_{1}$, then compute its time derivative by using (17)(28),

$$
\begin{aligned}
\dot{V}_{1} & =\left(\left|z_{1}\right|-\delta_{1}\right)^{n} f_{1}\left(z_{1}\right) s g_{1}\left(z_{1}\right) \dot{z}_{1} \\
& \leq-\left(c_{1}+k\right)\left(\left|z_{1}\right|-\delta_{1}\right)^{2 n} f_{1}\left(z_{1}\right)+\left(\left|z_{1}\right|-\delta_{1}\right)^{n}\left(\left|z_{2}\right|-\delta_{2}-1\right) f_{1}\left(z_{1}\right)
\end{aligned}
$$

Step 2: We choose a positive-definition function $V_{1}$ as

$$
V_{2}=V_{1}+\frac{1}{n}\left(\left|z_{2}\right|-\delta_{2}\right)^{n} f_{2}\left(z_{2}\right)
$$

and design virtual controller $\alpha_{2}$ as

$$
\alpha_{2}=-\left(c_{2}+k+1\right)\left(\left|z_{2}\right|-\delta_{2}\right)^{n-1} s_{2}\left(z_{2}\right)+\dot{\alpha}_{1}-\left(\delta_{3}+1\right) s g_{2}\left(z_{2}\right)
$$

with a positive design parameter $c_{2}$, then compute its time derivative,

$$
\begin{aligned}
\dot{V}_{2} \leq & -\sum_{i=1}^{2} c_{i}\left(\left|z_{i}\right|-\delta_{i}\right)^{2(n-i+1)} f_{i}\left(z_{i}\right)-k\left(\left|z_{1}\right|-\delta_{1}\right)^{2 n} f_{1}\left(z_{1}\right)+\left(\left|z_{1}\right|-\delta_{1}\right)^{n}\left(\left|z_{2}\right|-\delta_{2}-1\right) f_{1}\left(z_{1}\right) \\
& -\left(\left|z_{2}\right|-\delta_{2}\right)^{2(n-1)} f_{2}\left(z_{2}\right)+\left(\left|z_{2}\right|-\delta_{2}\right)^{n-1}\left(\left|z_{3}\right|-\delta_{3}-1\right) f_{2}\left(z_{2}\right)
\end{aligned}
$$

By using inequality $2 a b \leq a^{2}+b^{2}$, we have

$$
\begin{aligned}
\dot{V}_{2} \leq & -\sum_{i=1}^{2} c_{i}\left(\left|z_{i}\right|-\delta_{i}\right)^{2(n-i+1)} f_{i}\left(z_{i}\right)+\frac{1}{4 k}\left(\left|z_{2}\right|-\delta_{2}-1\right)^{2} \\
& -\left(\left|z_{2}\right|-\delta_{2}\right)^{2(n-1)} f_{2}\left(z_{2}\right)+\left(\left|z_{2}\right|-\delta_{2}\right)^{n-1}\left(\left|z_{3}\right|-\delta_{3}-1\right) f_{2}\left(z_{2}\right)
\end{aligned}
$$

for both cases $\left|z_{2}\right| \geq \delta_{2}+1$ and $\left|z_{2}\right|<\delta_{2}+1$, we can conclude that

$$
\dot{V}_{2} \leq-\sum_{i=1}^{2} c_{i}\left(\left|z_{i}\right|-\delta_{i}\right)^{2(n-i+1)} f_{i}\left(z_{i}\right)+\left(\left|z_{2}\right|-\delta_{2}\right)^{n-1}\left(\left|z_{3}\right|-\delta_{3}-1\right) f_{2}\left(z_{2}\right)
$$

Step n: Following this procedure step by step, we can derive the real control

$$
\begin{aligned}
& v(t)=\hat{\phi}(t) v_{1}(t) \\
& v_{1}(t)=-\left(c_{n}+1\right)\left(\left|z_{n}\right|-\delta_{n}\right) s g_{n}\left(z_{n}\right)-\hat{\mathbf{a}}^{T} Y-s g_{n}\left(z_{n}\right) \hat{M}+x_{d}^{(n)}+\dot{\alpha}_{n-1} \\
& \dot{\hat{\phi}}(t)=-\eta v_{1}(t)\left(\left|z_{n}\right|-\delta_{n}\right) f_{n} s g_{n}\left(z_{n}\right) \\
& \dot{\hat{\mathbf{a}}}(t)=\Gamma Y\left(\left|z_{n}\right|-\delta_{n}\right) f_{n} s g_{n}\left(z_{n}\right) \\
& \dot{M}(t)=\gamma\left(\left|z_{n}\right|-\delta_{n}\right) f_{n}
\end{aligned}
$$


where $\alpha_{n-1}$ can be obtained from the common form of virtual controllers $\alpha_{i}=-\left(c_{i}+k+1\right)\left(\left|z_{i}\right|-\delta_{i}\right)^{n-i+1} \operatorname{sg}_{i}\left(z_{i}\right)+\dot{\alpha}_{i-1}-\left(\delta_{i+1}+1\right) \operatorname{sg}_{i}\left(z_{i}\right), \quad(i=3, \cdots, n-1) \quad$ with positive design parameters $c_{i}$.

We define a positive-definition function as

$$
V=\sum_{i=1}^{n} \frac{1}{n-i+2}\left(\left|z_{i}\right|-\delta_{i}\right)^{(n-i+2)} f_{i}\left(z_{i}\right)+\frac{1}{2} \tilde{\mathbf{a}}^{T} \Gamma^{-1} \tilde{\mathbf{a}}+\frac{b_{p}}{2 \eta} \tilde{\phi}^{2}+\frac{1}{2 \gamma} \tilde{M}^{2}
$$

and compute its time derivative by using (13), (28), (30) and (32),

$$
\begin{aligned}
\dot{V}= & \dot{V}_{n-1}+\left(\left|z_{n}\right|-\delta_{n}\right)^{2} f_{n}\left(z_{n}\right) s g_{n}\left(z_{n}\right) \dot{z}_{n}+\tilde{\mathbf{a}}^{T} \Gamma^{-1} \dot{\tilde{\mathbf{a}}}+\frac{b_{p}}{\eta} \tilde{\phi} \dot{\tilde{\phi}}+\frac{1}{\gamma} \tilde{M} \dot{\tilde{M}} \\
\leq & -\sum_{i=1}^{n} c_{i}\left(\left|z_{i}\right|-\delta_{i}\right)^{2(n-i+1)} f_{i}\left(z_{i}\right)+\tilde{\mathbf{a}}^{T} \Gamma^{-1}\left(\Gamma Y z_{n}-\dot{\hat{\mathbf{a}}}\right) \\
& -\frac{b_{p}}{\eta} \tilde{\phi}\left(\eta v_{1} z_{n}+\dot{\tilde{\phi}}\right)-\left|z_{n}\right| \hat{M}+\left|z_{n}\right|\left|d_{b}[v](t)\right|+\frac{1}{\gamma} \tilde{M} \dot{\tilde{M}} \\
\leq & -\sum_{i=1}^{n} c_{i}\left(\left|z_{i}\right|-\delta_{i}\right)^{2(n-i+1)} f_{i}\left(z_{i}\right)+\tilde{\mathbf{a}}^{T} \Gamma^{-1}\left(\Gamma Y z_{n}-\dot{\hat{\mathbf{a}}}\right)-\frac{b_{p}}{\eta} \tilde{\phi}\left(\eta v_{1} z_{n}+\dot{\hat{\phi}}\right)+\frac{1}{\gamma} \tilde{M}\left(\gamma\left|z_{n}\right|-\dot{\hat{M}}\right) \\
= & -\sum_{i=1}^{n} c_{i}\left(\left|z_{i}\right|-\delta_{i}\right)^{2(n-i+1)} f_{i}\left(z_{i}\right)
\end{aligned}
$$

Thus we proved the first statement of the theorem. The rest of the statements can be easily proved following those of the proof of theorem 1, hence omitted here for saving space.

Remark 5: It is now clear the two proposed control schemes to mitigate the hysteresis nonlinearities can be applied to many systems and may not necessarily be limited to the system (2). However, we should emphasize that our goal is to show the fusion of the hysteresis model with available control techniques in a simpler setting that reveals its essential features.

\section{Simulation results}

In this section, we illustrate the methodologies presented in the previous sections using a simple nonlinear systems (Su, et al, 2000; Zhou et al, 2004) described by

$$
\dot{x}=a \frac{1-e^{-x(t)}}{1+e^{-x(t)}}+b w(t)
$$

where $w$ represents the output of the hysteresis nonlinearity. The actual parameter values are $a=1$, and $b=1$. Without control, i.e., $w(t)=0$, (33) is unstable, because $\dot{x}=\left(1-e^{-x(t)}\right) /\left(1+e^{-x(t)}\right)>0$ for $\quad x>0$, and $\dot{x}=\left(1-e^{-x(t)}\right) /\left(1+e^{-x(t)}\right)<0$ for $\quad x<0$. The objective is to control the system state $x$ to follow the desired trajectory $x_{d}=12.5 \sin (2.3 t)$. In the simulations, the robust adaptive control law (19) of Scheme I was used, taking $c_{1}=0.9, \gamma=0.2, \eta=0.1, \Gamma=0.1, \hat{\phi}(0)=0.8 / 3, \hat{M}(0)=2, \hat{x}(0)=3.05, v(0)=0, B_{1}=0.505$, 
$p(r)=e^{-6.7(0.1 r-1)^{2}}$ for $r \in(0,50]$. The simulation results presented in the Figure 3 is the comparison of system tracking errors for the proposed control Scheme I and the scenario without considering the effects of the hysteresis. For Scheme II, we choose the same initial values as before and $\delta=0.35$. The simulation results presented in the Figure 4 is the comparison of system tracking errors for the proposed control Scheme II and the scenario without considering the effects of the hysteresis. Clearly, the all simulation results verify our proposed schemes and show their effectiveness.

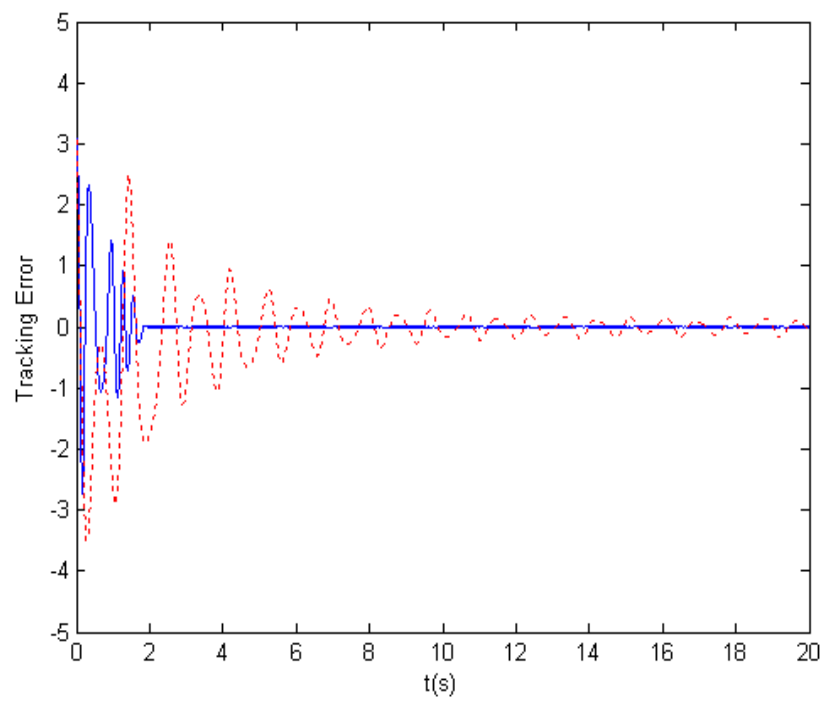

Fig. 3. Tracking errors -- control Scheme I (solid line) and the scenario without considering hysteresis effects (dotted line)

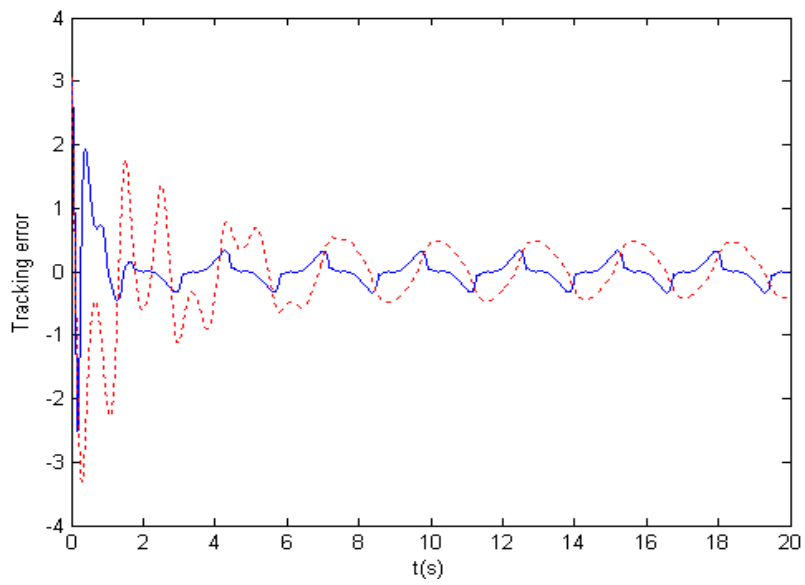

Fig. 4. Tracking errors -- control Scheme II (solid line) and the scenario without considering hysteresis effects (dotted line) 


\section{Conclusion}

We have for the first time constructed a class of new hysteresis model based on play-like operators and named it Prandtl-Ishlinshii-Like model where the play-like operators play a role of building blocks. We have proposed two control schemes to accomplish robust adaptive control tasks for a class of nonlinear systems preceded by Prandtl-Ishlinshii-Like models to not only ensure stabilization and tracking of the hysteretic dynamic nonlinear systems, but also derive the transient performance in terms of $L_{2}$ norm of tracking error as an explicit function of design parameters. By proposing Prandtl-Ishlinshii-Like model and using the backstepping technique, this paper has address a challenge that how to fuse a suitable hysteresis model with available robust adaptive techniques to mitigate the effects of hysteresis avoid constructing a complicated inverse operator of the hysteresis model. After this preliminary result, the idea in this paper is being further explored to deal with a class of perturbed strict-feedback nonlinear systems with unknown control directions preceded by this new hysteresis model.

\section{Acknowledgement}

This work was supported by the NSERC Grant, the National Natural Science Foundation of China (61004009, 61020106003), Doctoral Fund of Ministry of Education of China (20100042120033), and the Fundamental Research Funds for the Central Universities (N100408004, N100708001).

\section{References}

Su, C. Y.; Stepanenko, Y.; Svoboda, J. \& Leung, T. P. (2000). Robust Adaptive Control of a Class of Nonlinear Systems with Unknown Backlash-Like Hysteresis, IEEE Transactions on Automatic Control, Vol. 45, No. 12, pp. 2427-2432.

Fu, J.; Xie, W. F. \& Su, C. Y. (2007). Practically Adaptive Output Tracking Control of Inherently Nonlinear Systems Proceeded by Unknown Hysteresis, Proc. of the 46th IEEE Conference on Decision and Control, 1326-1331, New Orleans, LA, USA.

Banks, H. T. \& Smith, R. C. (2000). Hysteresis modeling in smart material systems, J. Appl. Mech. Eng, Vol. 5, pp. 31-45.

Tan, X. \& Baras, J. S. (2004). Modelling and control of hysteresis in magnetostrictive actuators, Automatica, Vol. 40, No. 9, pp. 1469-1480.

Tao G. \& Kokotovic P. V. (1995). Adaptive control of Plants with Unknown Hysteresis, IEEE Transactions on Automatic Control, Vol. 40, No. 2, pp. 200-212.

Tao G. \& Lewis, F., (2001). Eds, Adaptive control of nonsmooth dynamic systems, New York: Springer-Verlag, 2001.

Zhou J.; Wen C. \& Zhang Y. (2004). Adaptive backstepping control of a class of uncertain nonlinear systems with unknown backlash-like hysteresis, IEEE Transactions on Automatic Control, Vol. 49, No. 10, pp. 1751-1757.

Wen C. \& Zhou J.(2007). Decentralized adaptive stabilization in the presence of unknown backlash-like hysteresis, Automatica, Vol. 43, No. 3, pp. 426-440.

Tan X. \& Baras J. (2005). Adaptive Identification and Control of Hysteresis in Smart Materials, IEEE Transactions on Automatic Control, Vol. 50, No. 6, pp. 827-839. 
Iyer R.; Tan X., \& Krishnaprasad P.(2005), Approximate Inversion of the Preisach Hysteresis Operator with Application to Control of Smart Actuators, IEEE Transactions on Automatic Control, Vol. 50, No. 6, pp. 798-810.

Tan X. \& Bennani O. (2008). Fast Inverse Compensation of Preisach-Type Hysteresis Operators Using Field-Programmable Gate Arrays, in Proceedings of the American Control Conference, Seattle, USA, pp. 2365-2370.

Isidori A. (1989). Nonlienar Control Systems: an Introduction, 2nd ed. Berlin, Germany: Springer-Verlag.

Krasnoskl'skii M. A. \& Pokrovskii A. V. (1983). Systems with Hysteresis. Moscow, Russia: Nauka.

Brokate, M. \& Sprekels, J. (1996). Hysteresis and Phase Transitions, New York: SpringerVerlag.

Krejci P. (1996) Hysteresis, convexity and dissipation in hyperbolic equations, Gakuto Int. Series Math. Sci. \& Appl. Vol. 8, Gakkotosho, Tokyo.

Ekanayake D. \& Iyer V. (2008), Study of a Play-like Operator, Physica B: Condensed Matter, Vol. 403, No.2-3, pp. 456-459.

Krisic M.; Kanellakopoulos I. \& Kokotovic P. (1995). Nonlinear and Adaptive Control Design. New York: Wiley. 


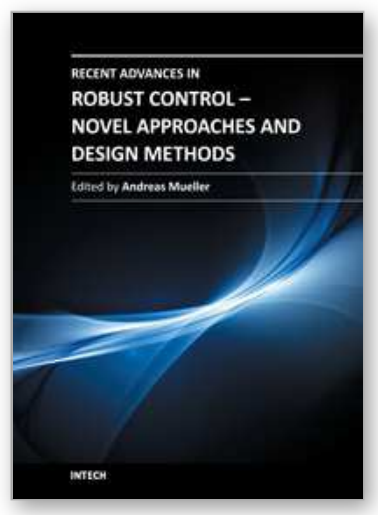

\author{
Recent Advances in Robust Control - Novel Approaches and \\ Design Methods \\ Edited by Dr. Andreas Mueller
}

ISBN 978-953-307-339-2

Hard cover, 462 pages

Publisher InTech

Published online 07, November, 2011

Published in print edition November, 2011

Robust control has been a topic of active research in the last three decades culminating in $\mathrm{H}$ 2/H_linfty and Imu design methods followed by research on parametric robustness, initially motivated by Kharitonov's theorem, the extension to non-linear time delay systems, and other more recent methods. The two volumes of Recent Advances in Robust Control give a selective overview of recent theoretical developments and present selected application examples. The volumes comprise 39 contributions covering various theoretical aspects as well as different application areas. The first volume covers selected problems in the theory of robust control and its application to robotic and electromechanical systems. The second volume is dedicated to special topics in robust control and problem specific solutions. Recent Advances in Robust Control will be a valuable reference for those interested in the recent theoretical advances and for researchers working in the broad field of robotics and mechatronics.

\title{
How to reference
}

In order to correctly reference this scholarly work, feel free to copy and paste the following:

Jun Fu, Wen-Fang Xie, Shao-Ping Wang and Ying Jin (2011). Robust Control of Nonlinear Systems with Hysteresis Based on Play-Like Operators, Recent Advances in Robust Control - Novel Approaches and Design Methods, Dr. Andreas Mueller (Ed.), ISBN: 978-953-307-339-2, InTech, Available from:

http://www.intechopen.com/books/recent-advances-in-robust-control-novel-approaches-and-designmethods/robust-control-of-nonlinear-systems-with-hysteresis-based-on-play-like-operators

\section{INTECH}

open science | open minds

\section{InTech Europe}

University Campus STeP Ri

Slavka Krautzeka 83/A

51000 Rijeka, Croatia

Phone: +385 (51) 770447

Fax: +385 (51) 686166

www.intechopen.com

\section{InTech China}

Unit 405, Office Block, Hotel Equatorial Shanghai

No.65, Yan An Road (West), Shanghai, 200040, China

中国上海市延安西路65号上海国际贵都大饭店办公楼 405 单元

Phone: +86-21-62489820

Fax: $+86-21-62489821$ 
(C) 2011 The Author(s). Licensee IntechOpen. This is an open access article distributed under the terms of the Creative Commons Attribution 3.0 License, which permits unrestricted use, distribution, and reproduction in any medium, provided the original work is properly cited. 